\title{
Fluctuating initial conditions in hydrodynamics for two-particle correlations
}

\author{
Y. Hama ${ }^{1 *}$ R.P.G. Andrade ${ }^{1}$, F. Grassi ${ }^{1}$ and W.-L. Qian ${ }^{2}$ \\ ${ }^{1}$ Instituto de Física, Universidade de São Paulo, Brazil \\ ${ }^{2}$ Departamento de Física, Universidade Federal de Ouro Preto, Ouro Preto-MG, Brazil \\ DOI: http://dx.doi.org/10.5689/UA-PROC-2010-09/17
}

\begin{abstract}
Event-by-event hydrodynamics, with fluctuating initial conditions, has shown to nicely reproduce several features of experimentally observed quantities in high-energy nuclear collisions. Here we discuss how it may help to understand, in a unified way, the various structures observed in the long-range two-particle correlations, both in nucleus-nucleus and $p-p$ collisions. Suggestions of how experimentally this description could be tested are also discussed.
\end{abstract}

\section{Introduction}

In hydrodynamic approach of nuclear collisions, it is assumed that, after a complex process involving microscopic collisions of nuclear constituents, at a certain early instant a hot and dense matter is formed, which would be in local thermal equilibrium. This state is characterized by some initial conditions (IC), usually parametrized as smooth distributions of thermodynamic quantities and four-velocity (see, for instance, $[1,2]$ ).

However, since our systems are small, important event-by-event fluctuations are expected in real collisions. Also, if the thermalization is verified at very early time, they should be very bumpy. In previous works, we introduced fluctuating $I C$ in hydrodynamics [3, 4], by using NEXUS event generator [5], and showed important effects on several observables.

In this paper, we briefly survey some of the previous results $[3,6,8,9]$ and then discuss more recent results on long-range two-particle correlations.

\section{Some consequences of fluctuating initial conditions}

In Figure 1, we show the energy-density distribution in a typical event of fluctuating IC, generated by NeXuS [5] for a central $\mathrm{Au}+\mathrm{Au}$ collision at $200 \mathrm{~A} \mathrm{GeV}$. Observe that the distribution is very bumpy, as expected in real collisions, having a tubular structure in $\eta$ (leftover from initial particle collisions).

Some consequences of such high-energy-density spots have been discussed in [6]. Because of high concentration of energy in small regions, each tube would suffer a violent explosion and, because of their small size, expand isotropically (in transverse directions). If such a tube is at

*Speaker 

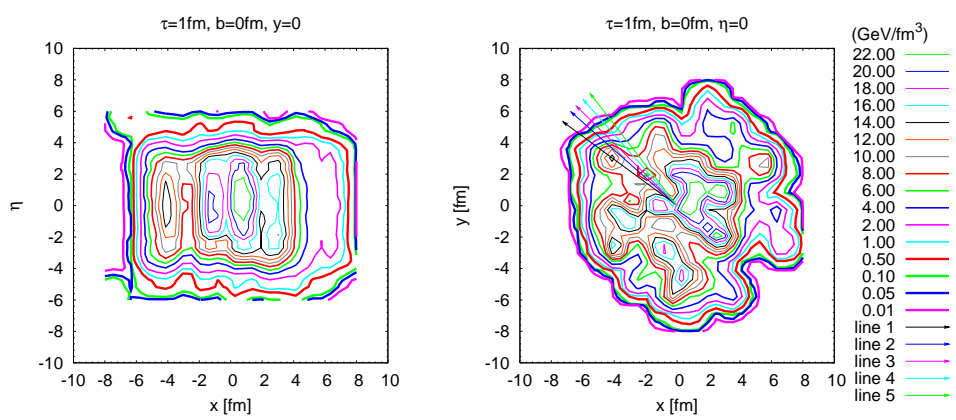

Figure 1: NEXUS Fluctuating Initial Conditions.

the surface of the matter, certainly the outgoing part of this initial acceleration would remain, producing high- $p_{T}$ particles, which would be isotropically distributed in the momentum space. Thus, first we expect that high- $p_{T}$ part of the $p_{T}$ spectra is enhanced when fluctuating IC are used in our computations, in comparison with the results with averaged (smooth) IC. In the second place, we expect that the elliptic flow coefficient $\left\langle v_{2}\right\rangle$ suffers reduction as we go to high- $p_{T}$ region, due to the additional high- $p_{T}$ isotropic components included now. A word of caution is necessary here. We are talking about effects of hot spots and not of the fluctuation, which makes $v_{2}$ coefficient larger in more central windows because it makes the eccentricity bigger [7]. As for the $\eta$ dependence of $v_{2}$, we know that the average matter density decreases as $|\eta|$ increases as reflected in the $\eta$ distribution of charged particles, so when such a blob is formed in the large- $|\eta|$ regions, its effects appear more enhanced. Therefore, we expect considerable reduction of $v_{2}$ in those regions. All these features have explicitly been verified in [6].

Another effect of small high-energy-density spots in the IC is manifested in the smaller HBT radii, as compared with the case of the smooth averaged IC. [9]. This has been shown both by using the Cooper-Frye prescription [10], and by the continuous-emission one [11].

Besides the effects of high-energy-density spots, fluctuations of IC imply evidently fluctuations of the resulting observable quantities. We have discussed that such fluctuations become quite large in the anisotropic-flow parameter $v_{2}[12,8]$, as has been effectively verified by experiments $[13,14]$.

\section{Two-particle correlations in hydrodynamic approach}

One of the most striking results in relativistic heavy-ion collisions is the existence of structures in the two-particle correlations $[15,16,17,18,19,20]$ plotted as function of the pseudorapidity difference $\Delta \eta$ and the angular spacing $\Delta \phi$. The so-called ridge has a narrow $\Delta \phi$ located around zero and a long $\Delta \eta$ extent. The other structure located opposite to the trigger has a single or double hump in $\Delta \phi$; its $\Delta \eta$ extent is not well established. More recently, the ridge structure has been observed also in $p p$ collisions at LHC [21].

In an earlier work, [22], we presented evidence that hydrodynamic approach reproduces all such structures in heavy-ion collisions. In [22], the events computed by using the hydrodynamic code SPheRIO [4], starting from event-by-event fluctuating IC, generated by NeXus [5], were analyzed in a similar way to the experimental ones, in particular the ZYAM method was used to remove effects of elliptic flow. We later developed a different method to remove elliptic flow from our data and checked that all structures are indeed exhibited and other features well 
reproduced (dependence on the trigger- or associated-particle transverse momentum, centrality, in-plane/out-of-plane trigger, etc) [23, 24, 25, 26].

\subsection{Mechanism of ridge formation - one-tube model}

As seen in Fig. 1, each NEXUS IC is very complicated, so difficult to visualize how various structures in the two-particle correlations are generated. In order to clarify the origin of the ridge structures, we introduced in [23] a simplified model which would allow to follow closely the time development of the fluid in the vicinity of one of the high-energy-density tubes. Evidently, only those tubes located close to the surface of the hot matter can contribute to the correlations. Thus, in our simplified model, we replace the complex bulk of the hot matter by the average over many events, leaving just one typical tube close to the surface, like the one on the line 1 of Fig. 1 , right. To simplify the computation, the longitudinal expansion is assumed boost-invariant and the transverse expansion is computed numerically (see details in [23]).

Figure 2 shows the temporal evolution of the hot matter in this model. As seen, pressed by the violent expansion of the high-energy-density tube, the otherwise isotropic radial flow of the background is deflected and guided into two well defined directions, symmetrical with respect to the initial tube position. Notice that the flow is clearly non-radial in these regions.
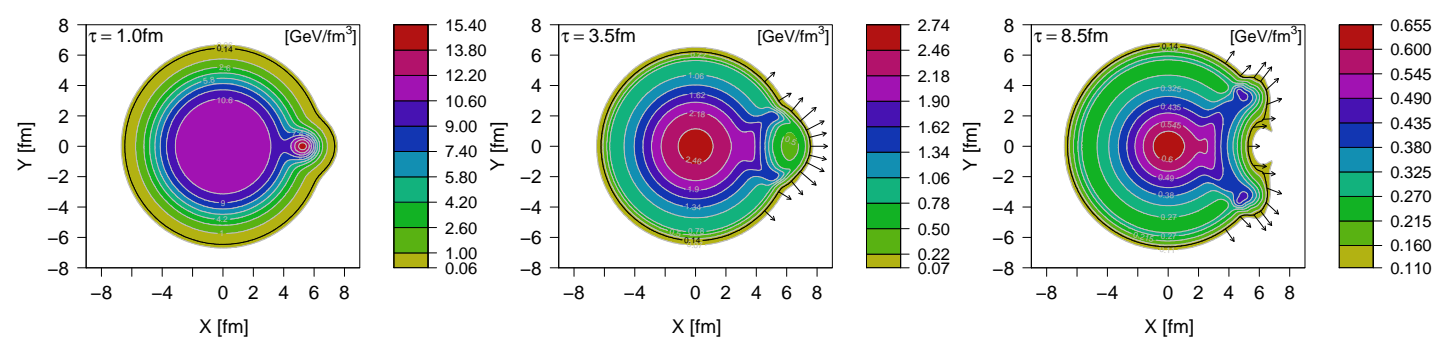

Figure 2: Temporal evolution of energy density for the one-tube model (times: 1.0, 3.5 and 8.5 $\mathrm{fm}$ ). Arrows indicate fluid velocity on the freeze out surface, thicker curve labeled by the freeze out temperature $0.14 \mathrm{GeV}$

The resultant single-particle angular distributions for two different $p_{T}$-intervals are plotted in Fig. 3, left. As expected they show symmetrical two-peak structures. From this plot, we can easily guess how the two-particle angular correlation will be. The trigger particle is more likely to be in one of the two peaks. We first choose the left-hand side peak. The associated particle is more likely to be also in this peak i.e. with $\Delta \phi=0$ or in the right-hand side peak with $\Delta \phi \sim+2$. If we choose the trigger particle in the right-hand side peak, the associated particle is more likely to be also in this peak i.e. with $\Delta \phi=0$ or in the left-hand side peak with $\Delta \phi \sim-2$. So the final two particle angular correlation must have a large central peak at $\Delta \phi=0$ and two smaller peaks respectively at $\Delta \phi \sim \pm 2$. Figure 3 (right) shows that this is indeed the case. The peak at $\Delta \phi=0$ corresponds to the near-side ridge and the peaks at $\Delta \phi \sim \pm 2$ form the double-hump ridge. We have checked that this structure is robust by studying the effect of the height and shape of the background, initial velocity, height, radius and location of the tube [23]. 

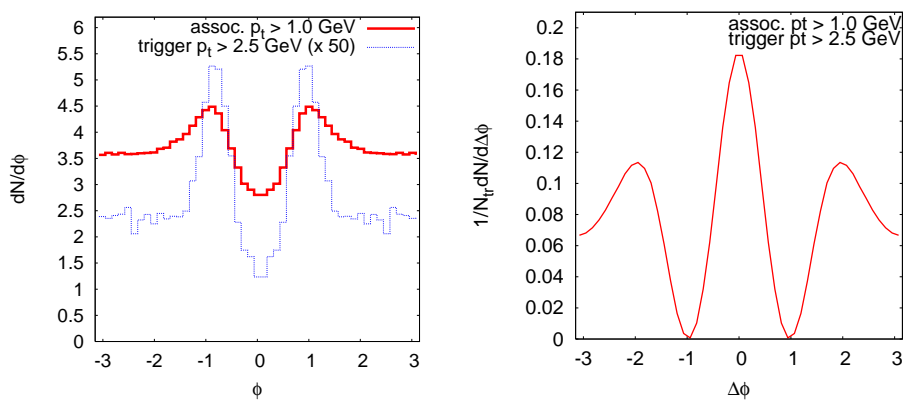

Figure 3: Angular distributions of particles in some different $p_{T}$ intervals (left) and resulting two-particle correlations (right), in the one-tube model.

As stressed above, this simplified description, namely one-tube boost-invariant model, has been introduced just to clarify the mechanism of ridge-structure formation. However, it is remarkable that this simple model can describe well so many characteristics observed in experiments. For a more realistic simulation, we should consider more complex events such as NeXus events and average over the fluctuations. For an event like the one shown in Fig. 1, only the outer tubes need to be considered. The shape of the two-particle correlations for a single tube (in particular the peak spacing) is relatively independent of its features so the various tubes will contribute with rather similar two-peaks emission pattern at various angles in the single-particle angular distribution. For this single event, the two-particle correlation has a well-defined main structure similar to that of a single tube (Fig. 3) surrounded by several other peaks and depressions due to trigger and associated particles coming from different tubes. These additional peaks and depressions have positions depending on the angle of the tubes between them. When averaging over many randomly fluctuating events these interference terms disappear and only the main one-tube like structure is left. The main advantage of this interpretation of ridge structures is that it involves essentially only the surface of the hot matter. The complexity of the kernel does not influence.

\subsection{Ridge in $p p$ collisions}

Durig this Symposium, new results on two-particle correlations in $p p$ collisions at LHC have been reported $[27,21]$. Accordingly, high-multiplicity $p p$ collisions show long-range $(\Delta \eta>4)$ nearside $(\Delta \phi \sim 0)$ ridge structure, which appears more enhanced when intermediate $p_{T}(1<$ $\left.p_{T}<3 \mathrm{GeV}\right)$ are considered.

Such a structure can be produced also within our tube-based description. However, since NeXuS generator [5] is based on nucleon-nucleon collisions as an ingredient, it can produce only one tube in the IC for $p p$ collisions themselves. Evidently, this will not produce ridges. Some parton based generator is needed to a more complete description of $p p$ data.

Here, we shall show how such a structure can be produced within our description, by considering a typical IC in $p p$, formed just by two tubes. Because of their small size, probably one-tube IC constitute the bulk of the fluctuating IC in high-energy $p p$ collisions. However, although in a small fraction, there should be also events with two tubes produced in the initial collisions of constituents. In Fig. 4, left, we show such two-tube IC. (K. Werner et al. discuss nearside ridge, by using EPOS generator [28], where the typical IC shown has a more ellip- 
tical shape. Although not necessarily, such IC can be obtained with two tubes with smaller distance.) It is easy to understand that the two tubes begin expanding radially, collide with each other, driving the flow into two opposite directions, as shown in Fig. 4, middle, producing an azimuthal distribution of particles with two peaks separated by $\pi$. This flow is similar to the elliptic flow, but probably with sharper peaks. Final results of two-particle correlations in such events are two ridges, one at $\Delta \phi \simeq 0$ and the other in the awayside at $\Delta \phi \simeq \pi$. In the CMS paper [21], the awayside ridge is not mentioned. In our opinion, this occurred because it is really not visible due to the dominance of jet originated correlations. It would be nice to experimentally separate the ridge from the jet events, by measuring the three-particle correlations in the following way:

Fix the first associated particle close to the trigger $\left(\Delta \phi_{1} \equiv \phi_{1}-\phi_{t} \sim \Delta \eta_{1} \equiv \eta_{1}-\eta_{t} \sim 0\right)$. Presumably, we are picking jets in this case. On the other side, if we fix $\Delta \phi_{1} \sim 0$ and $\Delta \eta_{1} \gtrsim$ 2 , we are selecting ridge particles. Then, measure the correlation with a second associated particle in the $\left(\Delta \phi_{2} \equiv \phi_{2}-\phi_{t}, \Delta \eta_{2} \equiv \eta_{2}-\eta_{t}\right)$ plane. We think that in the first case, we will see only typical jet correlations, with a high peak at $\Delta \eta \sim \Delta \phi \sim 0$ and a high associated ridge at $\Delta \phi_{2} \sim \pi$. And in the second case two low ridges separated by $\Delta \phi \sim \pi$, eventually with some contaminations of jet events. We call this kind of three-particle correlation $2+1$ correlation $[24,26]$.
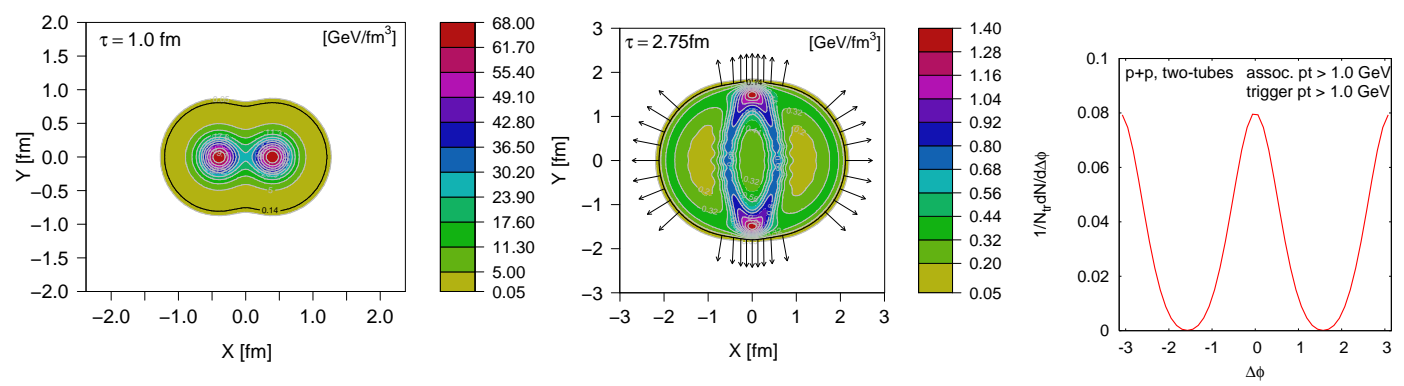

Figure 4: Time evolution of two-tube IC for $p p$ at two different instants $(\tau=1$ and $2.75 \mathrm{fm})$, and the resultant two-particle correlation as function of $\Delta \phi$. Here, the normalization has been done by using only one event, so the result should not be compared directly with data.

\section{Conclusions}

Hydrodynamic approach starting from event-by-event fluctuating initial conditions, with highenergy-density tubes, has shown to reproduce several features of experimentally observed quantities.

With regard to the long-range two-particle correlations, it gives a unified picture for the nearside and awayside structures as observed experimentally. In heavy-ion collisions, a highdensity tube located close to the surface of the hot matter divides the flow coming from inside into two currents, producing two-peak angular distribution. This two-peak distribution is the origin of both the nearside and the awayside ridges. In high-multiplicity $p p$ collisions, probably there are a fraction of events with two high-energy tubes. Such IC produce azimuthal distribu- 
tions with two peaks separated by $\pi$, resulting in a two-particle correlation with two opposite ridges, one observed as the nearside ridge and the other hindered below the awayside structure associated with jets.

See contribution by R. Andrade at this Symposium [29] for detailed discussion of the inplane/out-of-plane effect within one-tube model.

\section{Acknowledgments}

After the completion of the manuscript, we knew a paper [30], which discusses possible $v_{2}$ in $p p$ collisions at LHC by using a similar tube-based idea. We thank Dr. Bożek for bringing this information to our knowledge. We acknowledge funding from FAPESP and CNPq.

\section{References}

[1] T. Hirano and K. Tsuda, Phys. Rev. C 66 (2002) 054905.

[2] C. Nonaka and S.A. Bass, Phys. Rev. C 75 (2007) 014902.

[3] C.E. Aguiar, Y. Hama, T. Kodama and T. Osada, Nucl. Phys. A 698 (2002) 639c.

[4] Y. Hama, T. Kodama and O. Socolowski Jr., Braz. J. Phys. 35 (2005) 24.

[5] H.J. Drescher et al., Phys. Rev. C 65 (2002) 054902.

[6] R.P.G. Andrade, F. Grassi, Y. Hama, T. Kodama and W.-L. Qian, Phys. Rev. Lett. 101 (2008) 112301.

[7] B. Schenke, S. Jeon and C. Gale, arXiv: 1009.3244 [hep-ph].

[8] Y. Hama, R.P.G. Andrade, F. Grassi, W.-L. Qian, T. Osada, C.E. Aguiar and T. Kodama, Phys. Atom. Nucl. 71 (2008) 1558.

[9] O. Socolowski Jr., F. Grassi, Y. Hama and T. Kodama, Phys. Rev. Lett. 93 (2004) 182301.

[10] F. Cooper and G. Frye, Phys. Rev. D10 (1974) 186.

[11] F. Grassi, Y. Hama, and T. Kodama, Phys. Lett. B355(1995) 9; Z. Phys. C73 (1996) 153.

[12] C.E. Aguiar, Y. Hama, T. Kodama and T. Osada, Nucl. Phys. A698 (2002) 639c.

[13] P. Sorensen (for the STAR Collab.), J. Phys. G34 (2007) S897.

[14] C. Loizides (for the PHOBOS Collab.), J. Phys. G34 (2007) S907; B. Alver et al. (PHOBOS Collab.), Phys. Rev. Lett. 104 (2010) 142301.

[15] J. Putschke (for the STAR collaboration), Nucl. Phys. A783 (2007) 507.

[16] J. Putschke (for the STAR collaboration), J. Phys. G34 (2007) S679.

[17] M.P. McCumber (for the PHENIX Collaboration), J. Phys. G35 (2007) 104081.

[18] M.J. Horner (for the STAR Collaboration), J. Phys. G34 (2007) S995.

[19] E. Wenger (for the PHOBOS Collaboration), J. Phys. G35 (2008) 104080.

[20] B. Alver et al. (PHOBOS Collaboration), Phys. Rev. Lett. 104 (2010) 062301.

[21] CMS Collaboration, arXiv: 1009.4122 [hep-ex].

[22] J. Takahashi, B.M. Tavares, W.-L. Qian, R. Andrade, F. Grassi, Y. Hama, T. Kodama and N. Xu, Phys. Rev. Lett. 103 (2009) 242301.

[23] Y. Hama, R.P.G. Andrade, F. Grassi and W.-L. Qian, Nonlin. Phenom. Complex Sys. 12 (2010) 466.

[24] R.P.G. Andrade, F. Grassi, Y. Hama and W.-L. Qian, J. Phys. G37 (2010) 094043.

[25] R.P.G. Andrade, F. Grassi, Y. Hama and W.-L. Qian, arXiv: 1008.0139 [hep-ph]; to appear in Nucl. Phys. A.

[26] R.P.G. Andrade, F. Grassi, Y. Hama and W.-L. Qian, arXiv: 1008.4612 [nucl-th].

[27] X. Janssen (for the CMS Collab.), talk at this Symposium.

[28] K. Werner, Iu. Karpenko and T. Pierog, arXiv: 1011.0375 [hep-ph].

[29] Rone P.G. Andrade, Frédérique Grassi, Yogiro Hama and Wei-Liang Qian, in this Proceedings.

[30] P. Bożek, Acta Phys. Polon. B41 (2010) 837. 